\title{
TRAFFIC SIGN BOARD DETECTION AND RECOGNITION FOR AUTONOMOUS VEHICLES AND DRIVER ASSISTANCE SYSTEMS
}

\author{
Y.D. Chincholkar and Ayush Kumar \\ Department of Electronics and Telecommunication Engineering, Sinhgad College of Engineering, India
}

\begin{abstract}
In the recent year's many approaches have been made that uses image processing algorithms to detect traffic sign boards. Edge detection is used to avoid segmentation problems of the existing method. Color based segmentation faces the challenge of adaptive thresholding which fails in real time scenarios. This proposed algorithm is yet another approach to detect traffic sign boards from video sequences. The first step of this work is the pre-processing of the video frame which is achieved by the gray scale conversion and edge detection and the second step is the extraction of the objects. Hough Transform algorithm is then applied to measure properties of image regions for further analysis. The different feature points which include perimeter, area, filled area, solidity and centroid are extracted for the detection of the traffic sign board. Feature generation and classification are done on the recognition side to get the class of the detected object. The input for the project is video sequences taken from a camera placed on the vehicle.
\end{abstract}

\section{Keywords:}

Hough Transform, Machine Learning Algorithm, Traffic Detection, Feature Classification

\section{INTRODUCTION}

Traffic sign board detection and recognition system have many applications such as driver assistance, safety information to the drivers, automatic vehicles. There are many potential challenges concerning detection process because of the complex environment of the roads and scenes around them and there might be road signs present in different conditions. Implementation of the traffic sign board detection system using some processing board along with a camera mounted on the vehicles. Various algorithms have been used for detection of sign boards. Different kind of devices available which detects the traffic sign boards for the driving information. However, these gadgets fail to work in bad environmental condition. It might be the possibility that road sign boards are faded, or it may vary in different outdoor lightening condition. Polluted air and bad weather also influence the driving. Thus a traffic sign board detection and recognition system are desired for the driver safety which provides the safety information in the various driving environment before something may go wrong. Currently, there is no such system available which assists the driver with such information in any conditions. Nowadays of automation, this system will be beneficial to help the driver on real time with some safety information. It can also be used in road side maintenance. Currently, a health check of traffic sign boards is done manually by transportation authorities. By using this system, it can be automated.

The schematic representation of this work is shown in Fig.1.

The paper is organized as follows. The section 1 contains the applications and introduction. The literature survey is described in section 2. The proposed method is discussed in section 3. The section 4 presents the gray scale conversion used for background subtraction. Edge detection using canny operator is discussed in section 5. The extraction of traffic sign board object is described in section 6 . The section 7 describes the feature generation section 8 feature selection to classify the traffic sign boards from different classes from the database. The last section concludes this work.

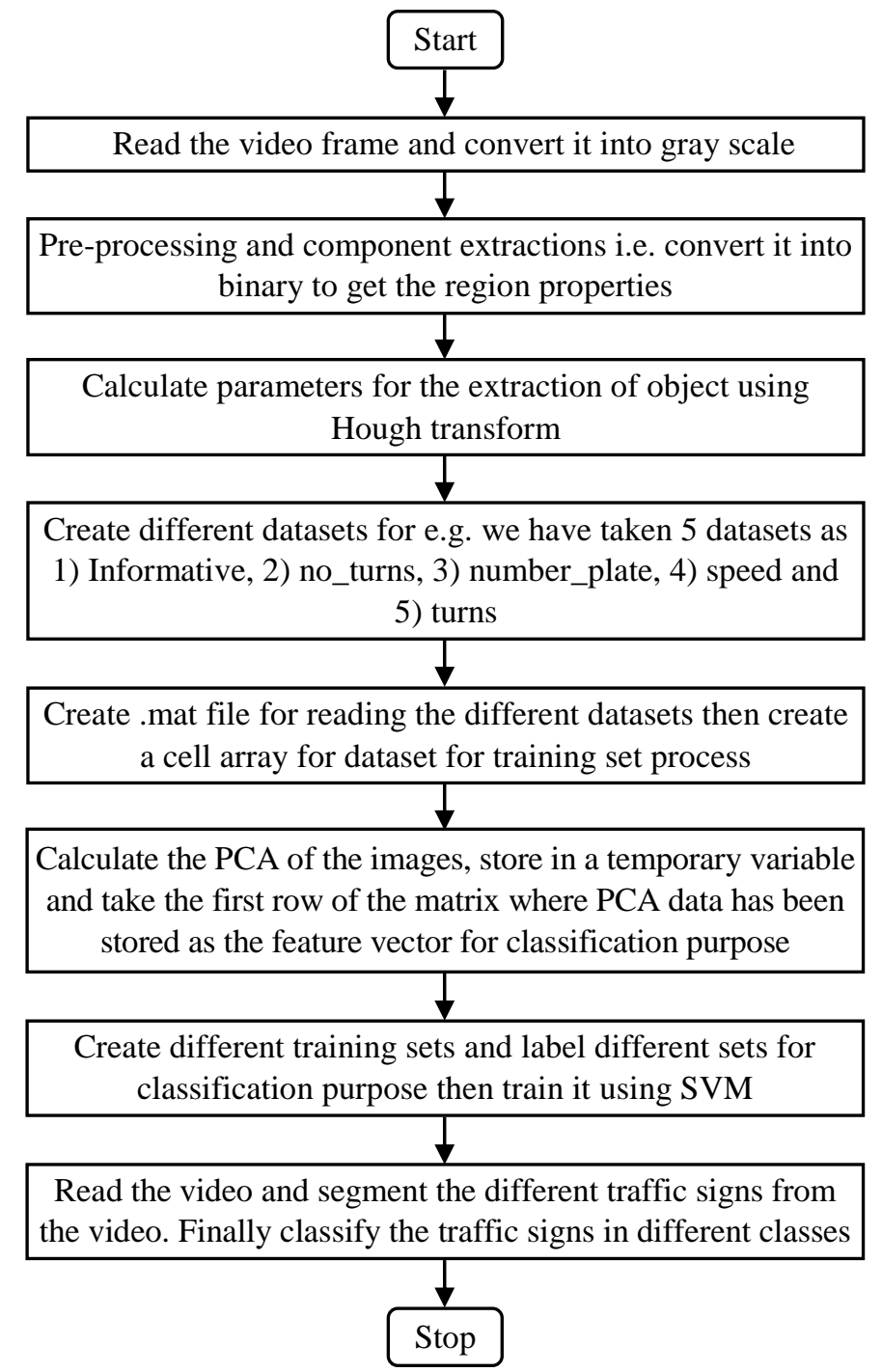

Fig.1. Schematic Representation of the proposed method

\subsection{LITERATURE SURVEY}

Hankizi [1] introduces a real-time traffic sign detection system with a camera mounted on a vehicle for a driver assistance system. The proposed recognition system includes feature generation and feature matching by all detected ROI's on the same scale to 
provide enough information in driver assistance function. A threestep detection that is preprocessing, color-based segmentation and shape feature based detection are performed to obtain the results. Evaluation of performance based on detection rate is done with the video sequences. Jung [2] used an artificial neural network algorithm for training and the classification. The present a traffic sign recognition system in real time with deep convolution that helped in advanced driver assistance to recognize the traffic sign board for more information on roads. The recognition framework accomplishes performance in real time for autonomous vehicles. Performance is assessed on precision parameter upon brightness condition, other environmental conditions. Moutarde [4] applied a rectangular detection method to detect the traffic sign board and then applied a neural network algorithm to recognize them.

Angela and Hua [5] furthermore used Hough-change for the disclosure of quadrilateral sign sheets. They used an incline based Hough change to recognize the edges in the image and a short time later close circuit ID and abundance line deletion technique to discover the sign board in the image. Loy and Barnes [6] displayed a figuring that recognizes polygon shaped signs (e.g., square, triangle, octagon) using extended symmetry identifier. The exactness of these shape develop procedures depends in light of a lot of the edges being used by them, and these edges are unprotected against uproarious pixels.

Sign Boards are planned to have particular symmetrical shapes. Various authors have made different figuring making usage of the symmetrical shape features of the sign boards. Zelinsky and Barnes [7] used the symmetry feature of the signs to perceive circuitous signs with a measure of winding symmetry in the image. In another paper, Barnes [9] used extended symmetry and standard polygon disclosure figuring consequent to diminishing the fuss in the images to improve the recognition happens. In any case, it is observed that these symmetry features of the sign board, when evaluated from the images, are clashing under different challenge changes and various observations. Sign board ID is furthermore performed using design organising procedures. However, since they are computationally exorbitant, they are associated with more small regions controlled by performing shading division previously using design planning [7]. A speedy arrangement planning system using imitated toughening figuring is proposed by Betke and Makris [10].

Features are learned for the essential parts of the perceived objects. Various computations exist in the literature work, which assessed invariant properties of the printed characters. For example, the geometric moment invariants deduced by $\mathrm{Hu}$ [8] are used as a piece of various optical character affirmation figuring. Zernike [9] proposed a course of action of minutes in light of Zernike polynomials. Rodriguez and Maudes [10] used the number of holes in the characters and their level and vertical projections as features. Wu et al. [11] proposed character affirmation figuring in the perspective of case mapping. They got the features from the histograms of the various parts of the characters. Hausdorff [12] expel, which is used to consider two twofold pictures, is furthermore used in character affirmation computations.

Moreover computations have been done in perspective of support vector machines [13] and arrange organizing [14] [15] frameworks. Neural framework [16] is another crucial strategy used for optical character affirmation in various applications.
Probabilistic neural frameworks procedure is used for seeing the letter sets what's more, numbers.

\section{PROPOSED METHOD}

There have been multiple approaches to detect the traffic sign board like color based segmentation. The existing approach to segment the region based on a color feature of the object faces the problem during real-time scenarios because it is dependent on adaptive thresholding. So previous methods results vary drastically as lighting and luminance condition changes. The morphological operations are performed to get better results by clearing the holes in the image with erosion and dilation. Due to background color variations and un-avoidable noise, this method is not optimum in real time scenarios.

Thus Hough transform approach is proposed to detect traffic sign boards. This algorithm is applied after edge detection. Hough Transform (HT) algorithm for detecting predefined shapes, has always been recognized as a unique, promising method for shape and motion analysis in images that contain noise, missing and extraneous data. To facilitate the target recognition and at the same time reduce the amount of information presented, images are transformed from gray level to the magnitude and direction of the gray level changes using edge operators.

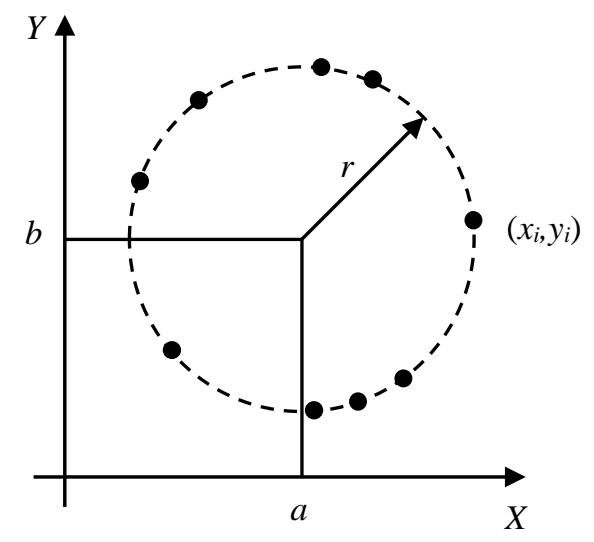

Fig.2. Circle in parametric space

When more than one circle coincides in parametric space with each other, then it's a strong circle in the original image. Much of the efficiency of the Hough Transform is dependent on the quality of the input data and the edges must be detected well for the Hough Transform to be efficient. The Canny edge detector is used before applying the Hough transform algorithm. It is used to identify a triangle and rectangle using Hough lines and to find the angle between them. The parametric space of traffic sign board is used to recognize it. Euclidean distance is calculated to get the traffic sign board in the parametric space. Radius is obtained to identify the circular traffic sign board in the algorithm.

\section{GRAY SCALE CONVERSION}

The road sign images are color RGB images, so the primary in function of the preprocessing algorithm is to convert color images to gray scale images for the faster operation. So, 24-bits RGB is converted to 8-bits gray scale image for easier processing in real time. The below experimental results shows the RGB 
image of road scene having traffic sign board and corresponding gray scale image. This results is obtained as first part of preprocessing, so that gray scaled image frame is processed for edge detection.
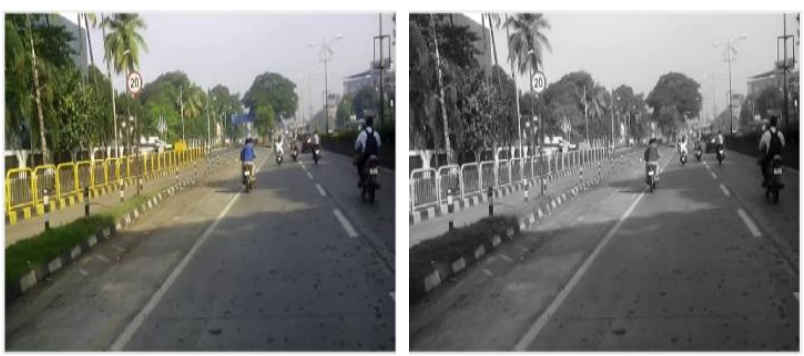

Fig.3. Color and Gray scale image

\section{EDGE DETECTION}

Edge detection is a kind of picture division procedures which decides the nearness of an edge or line in a picture and adequate blueprints for them. The principle motivation behind edge identification is to streamline the picture information with a specific end goal to limit the measure of information to be prepared. For the most part, an edge is characterized by the limit pixels that interface two separate locales with changing picture sufficiency properties. There are distinctive methodologies and calculation to discover the edge in picture preparing that, meanwhile, watchful administrator because of high precision and low handling volume has a more great execution contrasted with different calculations. The Canny edge detector is used for better accuracy and better performance. The below experiment is performed to get the edges from gray scaled image frame. The detected image frame is shown along with their input gray scaled image.

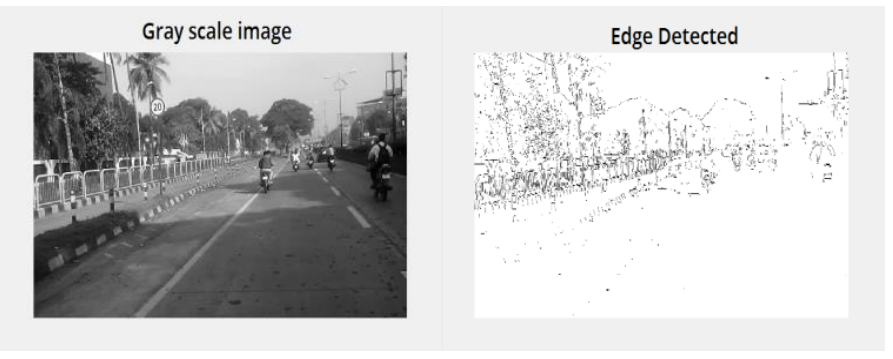

Fig.4. Gray scale image with Edge

\section{EXTRACTION OF TRAFFIC SIGN BOARD}

Hough Transform algorithm is used for the shape extraction for traffic sign board. This approach has a good detection rate with high accuracy for traffic sign boards in different traffic condition. The below results are obtained on Windows 7 operating system with 4GB RAM using a video sequence of road scenes.

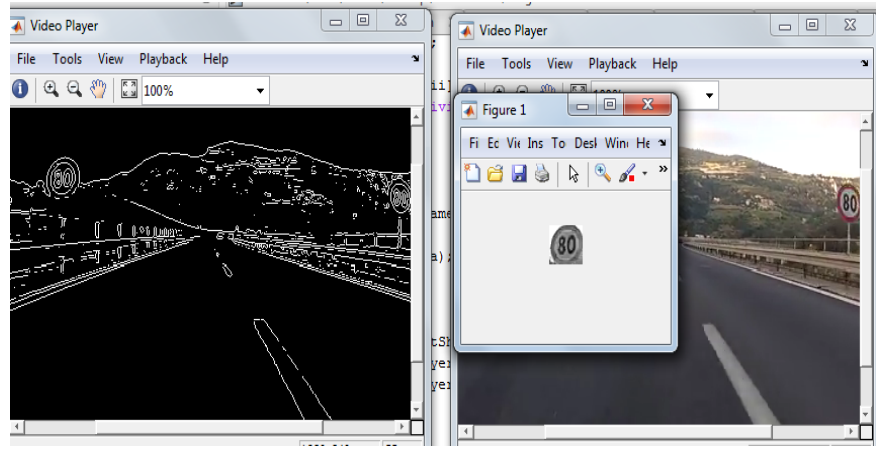

Fig.5. Traffic sign board Detection by Hough Transform

\section{FEATURE GENERATION}

Principal component analysis (PCA) is performed to get the primary component from the set of observations of correlated variables. The principal components are orthogonal because they are the Eigen vectors of the symmetric covariance matrix. The maximum possible value is obtained from the Eigen vector matrix which is the most substantial Eigen value of the matrix. Consider a data matrix, $X$, with column-wise zero empirical mean (the sample mean of each column has been shifted to zero), where each of the $n$ rows represents a different repetition of the experiment, and each of the $p$ columns gives a kind of feature (say, the results from a sensor). Mathematically the transformation is defined by a set of $p$ dimensional vectors of width or loadings $w(k)$ where $k$ varies from 1 to $p$ that maps each row vector $X(i)$ of $X$ to a new vector of principal component scores $t(i)$ where $i$ varies from 1 to $k$, given by Eq.(1) and Eq.(2).

$$
\begin{gathered}
t_{k(i)}=X_{(i)} \cdot W_{(k)} \\
W_{(i)}=\arg \max \left\{\frac{W^{T} X^{T} X_{W}}{W^{T} W}\right\}
\end{gathered}
$$

The quantity to be maximised can be recognised as a Rayleigh quotient. A standard result for a symmetric matrix such as $X^{T} X$ is that the quotient's maximum possible value is the largest eigenvalue of the matrix, which occurs when $w$ is the corresponding Eigen vector. The reduced dimension computational structure allows the relevant data characteristics to be identified with a little amount of information loss. After this analysis, we get the feature for classification.

\section{FEATURE CLASSIFICATION}

For the sorting, SVM classifier is used to classify the traffic sign board into the classes to assist the driver. SVM classifier has been selected for fast response in real time. The pro-per-response is obtained using SVM as Support vector machines are comparatively quicker and they classify binary based either match found or not found; there won't be any ambiguity in classification. MAT file has been prepared for each dataset class for the training set. The below MAT file obtained training set for No Turns class. 


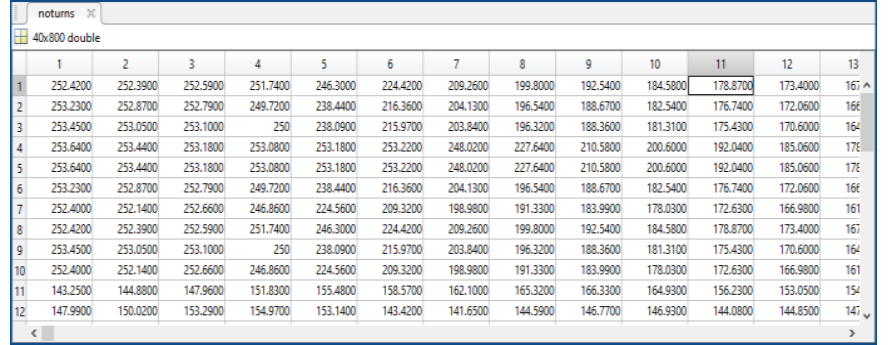

Fig.6. MAT file for a training set of no turn's class

The training set has been prepared for all the classes. Then we apply a machine learning algorithm that is the SVM classifier to get the detected traffic sign board with the class name. The class name could be informative, turns, stop, no turns etc. When the class of the traffic sign board has been found, it can be displayed on the system for driving assistance or for autonomous vehicles. The traffic sign board is shown along with the name of the class after comparing with the database with the help of a processing board and a camera mounted on a vehicle. The below results are obtained with the name of the dataset class on Matlab. The class name of the traffic sign board is shown to assist with information about the detected traffic sign board.

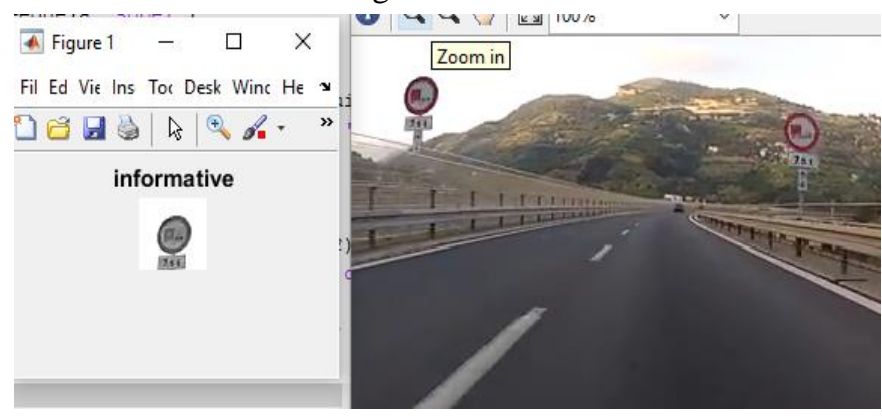

Fig.7. Results obtained after SVM classification

\section{RESULTS AND DISCUSSION}

The existing color based segmentation is applied to detect traffic sign boards, however it had the problem with R, G, B color that interfere the algorithm and the extraction of traffic sign boards is changed drastically. The below results describes the problem with color based segmentation when red plane interfere with this algorithm.

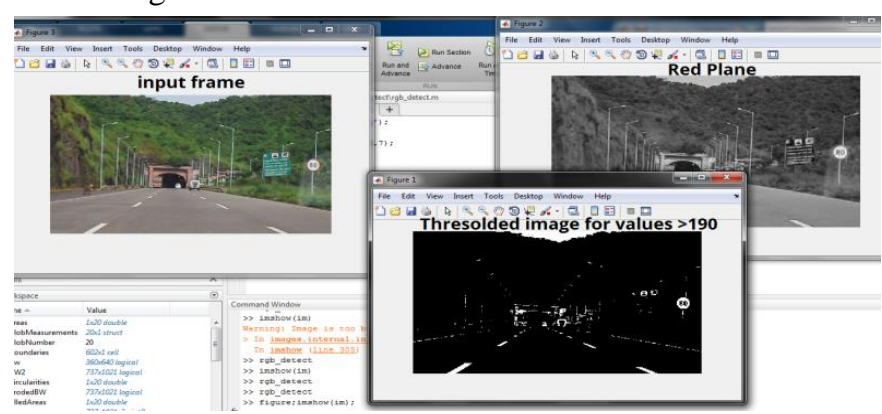

Fig.8. Traffic sign board Detection with existing approach of segmentation with adaptive thresholding

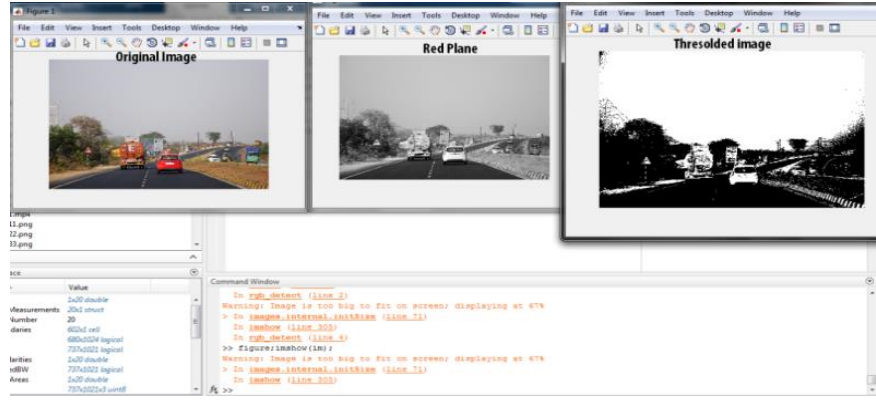

Fig.9. Traffic sign board Detection with existing approach of segmentation which is not appropriate in real time because red color interferes the algorithm

Later tried with adaptive thresholding in existing algorithm but it's very much affected with background variations and noise for real time scenarios. Because of that few major performance issue, existing extraction algorithm is not used for detection of traffic sign board from the desired system. The existing system is tested with 121 traffic sign boards frame then it is able to extract 78 traffic sign board images. The existing system with color based segmentation not optimum for detection. The system is implemented on laptop having I3 processor and 4GB RAM and another laptop to play the video sequences. Recognition is also not good if the traffic sign boards are not extracted accurately with efficiency. To overcome the problem with existing system with color based segmentation, Hough transform is proposed to extract traffic sign board after pre-processing steps they are gray scale conversion and edge detection. With this algorithm up to $98 \%$ detection rate is obtained over real time video sequences of 2.8 minutes of road scenes. It is also implemented with same system set up. Experimental results are shown below.

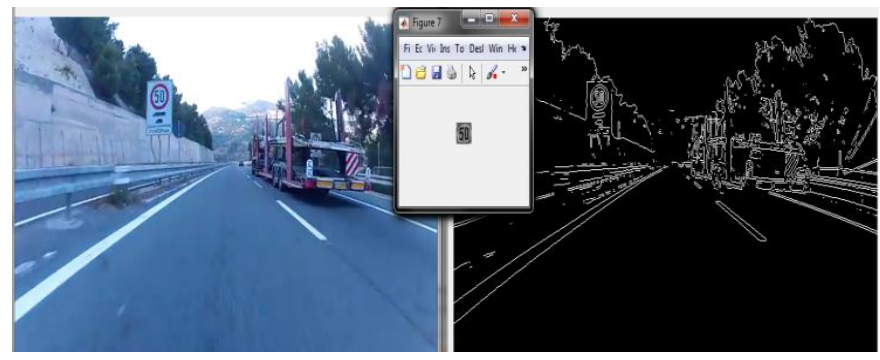

Fig.10. Traffic sign board Detection by Hough Transform

Then feature generation using PCM discussed in section 7 and feature classification using SVM classifier discussed in section 8, are applied after extraction of traffic sign boards. So it provides safety information for driver assistance with different class name as per saved in database. All the steps of this system development are discussed from section 3 to 8 of this paper with their results. Experimental results is obtained as below after combining all processes involved for this system development. 


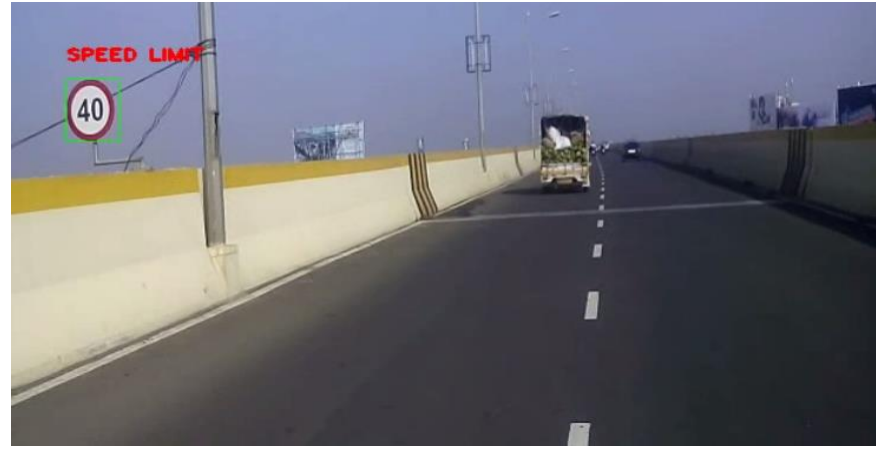

Fig.11. Traffic sign board Detection and recognition system result with safety information

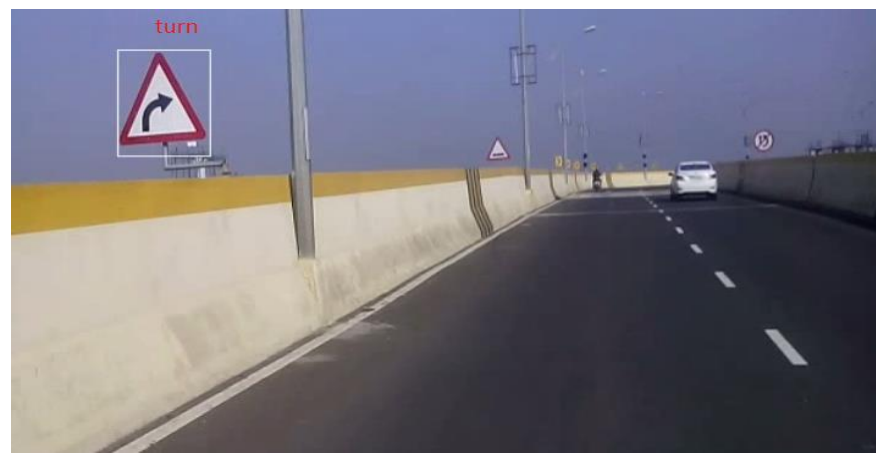

Fig.12. Traffic sign board Detection and recognition system result with assistance information

\section{CONCLUSION AND FUTURE WORK}

In this paper, the Hough transform algorithm is proposed based on the Euclidean distance over the existing color based segmentation approach to detect the traffic sign boards. Moreover, the experimental results prove that the proposed algorithm is efficient for real-time systems. The objective of this paper is to assist the driver in traffic conditions and warn him about the rules to reduce accidents. Traffic sign detection and recognition system have been developed which has excellent execution under testing conditions, for example, to a significant degree dim brightening, immersed foundation, fractional impediments and broad scale varieties. The proposed traffic sign board detection system can be used as a part of advanced driver assistance system to provide the safety information to the driver in real time. Also, it can be used for a driverless vehicle for the traffic sign board detection and recognition due to efficient detection with high accuracy. The system can be used for automated road maintenance due to high efficiency and detection rate. Future work can be carried Lane Identification and Warning system for the automatic vehicles.

\section{REFERENCES}

[1] Zhihui Zheng, Bo Wang and Zhifeng Gao, "Robust Traffic Sign Recognition and Tracking for Advanced Driver Assistance Systems", Proceedings of $15^{\text {th }}$ International IEEE Conference on Intelligent Transportation System, pp. 704-709, 2012.
[2] Seokwoo Jung, Unghui Lee, Jiwon Jung and David Hyunchul, "Real-Time Traffic Sign Recognition System with Deep Convolutional Neural Network", Proceedings of $13^{\text {th }}$ International Conference on Robotics and Ambient Intelligence, pp. 31-34, 2016.

[3] Rafael C. Gonzalez and Richard E. Woods, "Digital Image Processing", 2 ${ }^{\text {nd }}$ Edition, Pearson Education, 2004.

[4] F. Moutarde, A. Bargeton, A. Herbin and L. Chanussot, "Modular Traffic Signs Recognition Applied to On-VehicleTime Visual Detection of American and European Speedlimit Signs", Proceedings of IEEE International Symposium on Intelligent Vehicles, pp. 1122-1126, 2007.

[5] Angela Tam, Hua Shen, Liu and Xiaoou Tang, "Quadrilateral Signboard Detection and Text Extraction", Proceedings of the International Conference on Imaging Science, Systems and Technology, pp. 708-713, 2003.

[6] N. Barnes and G. Loy, "Fast Shape-Based Road Sign Detection for a Driver Assistance System. Intelligent Robots and Systems", Proceedings of the International Conference on Intelligent Robots and Systems, pp. 70-75, 2004.

[7] N. Barnes and A. Zelinsky, "Real-Time Radial Symmetry for Speed Sign Detection", Proceedings of International Symposium on Intelligent Vehicles, pp. 566-571, 2004.

[8] M.K. Hu, "Visual Pattern Recognition by Moment Invariants", IRE Transactions on Information Theory, Vol. 8, No. 2, pp. 179-187, 1962.

[9] F. Zernike, "Diffraction Theory of the Cutting Process and its Improved Shape, the Phase Contrast Method", Physica, Vol. 1, No. 7, pp. 689-704, 1934.

[10] Juan J. Rodriguez, Cesar Garcia-Osorio and Jesus Maudes, "License Plate Number Recognition-New Heuristics and A Comparative Study of Classifiers", Proceedings of International Conference on Informatics in Control, Automation and Robotics, pp. 268-273, 2008.

[11] Hsien-Chu Wu, Chwei-Shyong Tsai and Ching-Hao Lai, “A License Plate Recognition System in E-Government", Information and Security, Vol. 15, No. 2, pp. 199-210, 2004.

[12] F. Martin, M. Garcia and J.L. Alba, "New Methods for Automatic Reading of Vehicle License Plates", Proceedings of International Conference on Signal Processing, Pattern Recognition, and Applications, pp. 331-338, 2002.

[13] K.K. Kim, K.I. Kim, J.B. Kim and H.J. Kim, "LearningBased Approach, for LicensePlate Recognition", Proceedings of IEEE Signal Processing Society Workshop on Neural Networks for Signal Processing, pp. 614-623, 2000.

[14] P. Comelli, P. Ferragina, M.N. Granieri and F. Stabile, "Optical Recognition of Motor Vehicle License Plates", IEEE Transactions on Vehicular Technology, Vol. 44, No. 4, pp. 790-799, 1995.

[15] Yo Ping Huang, Shi-Yong Lai and Wei-Po Chuang, "A Template-Based Model for License Plate Recognition”, Proceedings of International Conference on Networking, Sensing and Control, pp. 737-742, 2004.

[16] Tran Duc Duan, Tran Le Hong Du, Tran Vinh Phuoc and Nguyen Viet Hoang, "Building an Automatic Vehicle License-Plate Recognition System”, Proceedings of International Conference on Computer Science, pp. 59-63, 2005. 
[17] GiYeong Bae, JeongMok Ha, Jea Young Jeon, Sung Yong Jo and Hong Jeong, "LED Traffic Sign Detection using Rectangular Hough Transform", Proceedings of International Conference on Information Science and Applications, pp. 1-4, 2014.

[18] Seokwoo Jung, Unghui Lee, Jiwan Jung and David Hyunchul Shim, "Real Time Traffic Sign Board Recognition with Deep Convolutional Neural Network", Proceedings of International Conference on Ubiquitous Robots and Ambient Intelligence, pp. 23-28, 2016.
[19] Zhihui Zheng, Hanxizi Zhang, Bo Wang and Zhifeng Gao, "Robust Traffic Sign Recognition and Tracking for Advanced Driver Assistance Systems", Proceedings of $15^{\text {th }}$ International Conference on Intelligent Transportation Systems, pp. 704-709, 2012.

[20] R. Thendral, A, Suhasini and N. Senthil, "A Comparative Study of Color and Edge based Segmentation", Proceedings of International Conference on Communication and Signal Processing, pp. 463-466, 2014. 